\title{
ULTRAVIOLET STUDIES OF NOVAE
}

\author{
M. Friedjung \\ and
}

B. Rocca

Institut d'Astrophysique de Paris

High dispersion spectra of WZ Sagittae and Nova Cygn1 1978, taken by IUE, have been studied by us.

Of the two spectra of WZ Sagittae in our possession, the long wave region one shows little besides the interstellar $\mathrm{Mg}$ II doublet. The more interesting short wavelength region has relatively wide $(130-250 \mathrm{~km} / \mathrm{s})$ resonance absorption lines sometimes at the center of wider emisstion. No clear sign of ejection is seen. The study of the profiles of the CIV, NV, OI, and SiIV resonance lines and of HeII $1640 \AA$ Indicates:

a) The absorption lines are probably not interstellar (widths, depths).

b) A standard accretion disk model can be used. The high Ionization lines are probably formed in a region ionized by the boundary layer. The line width indlcates that they are formed near the edge of the disk (top of raised edge?), illuminated by radiation from a radius a factor of 10 smaller.

A long wave region high dispersion spectrum of Nova Cygni 1978 has also been studied. Equivalent widths of the lines of multiplets 1,2 , and 3 of FeII and of multiplet I of MnII were measured. These Iines are in the same velocity system as that of the strong MgII IInes described by Cassatella et al. (A and A, 74, L18) blue shifted about $80 \mathrm{~km} / \mathrm{s}$ from a narrow interstellar line, and attributed by them to gas existing before the outburst. A study of the curve of growth indicates conditions similar to those of interstellar gas, the excited FeII lines at $385 \mathrm{~cm}^{-1}$ having a lower level population/g of $\geq 1.5$ dex less than that for the unexcited lines. Interpreted as an excitation temperature this would correspond to $\leq 160^{\circ}$. The MnII/ FeII colum density ratio of $1 / 6$ is exceptionally high. It is still not certain whether the gas is interstellar or circumstellar. 